\title{
A phase 1 'window-of-opportunity' trial testing evofosfamide (TH-302), a tumour-selective hypoxia-activated cytotoxic prodrug, with preoperative chemoradiotherapy in oesophageal adenocarcinoma patients
}

\author{
Ruben T. H. M. Larue ${ }^{1 \dagger}$, Lien Van De Voorde ${ }^{1 * \dagger}$, Maaike Berbée ${ }^{1}$, Wouter J. C. van Elmpt ${ }^{1}$, Ludwig J. Dubois ${ }^{1}$, \\ Kranthi M. Panth1, Sarah G. J. A. Peeters ${ }^{1,2}$, Ann Claessens ${ }^{1}$, Wendy M. J. Schreurs ${ }^{3}$, Marius Nap ${ }^{4}$, \\ Fabiënne A. R. M. Warmerdam ${ }^{5}$, Frans L. G. Erdkamp ${ }^{5}$, Meindert N. Sosef ${ }^{6,7}$ and Philippe Lambin ${ }^{1}$
}

\begin{abstract}
Background: Neo-adjuvant chemoradiotherapy followed by surgery is the standard treatment with curative intent for oesophageal cancer patients, with 5-year overall survival rates up to $50 \%$. However, patients' quality of life is severely compromised by oesophagectomy, and eventually many patients die due to metastatic disease. Most solid tumours, including oesophageal cancer, contain hypoxic regions that are more resistant to chemoradiotherapy. The hypoxia-activated prodrug evofosfamide works as a DNA-alkylating agent under these hypoxic conditions, which directly kills hypoxic cancer cells and potentially minimizes resistance to conventional therapy. This drug has shown promising results in several clinical studies when combined with chemotherapy. Therefore, in this phase I study we investigate the safety of evofosfamide added to the chemoradiotherapy treatment of oesophageal cancer.

Methods/Design: A phase I, non-randomized, single-centre, open-label, $3+3$ trial with repeated hypoxia PET imaging, will test the safety of evofosfamide in combination with neo-adjuvant chemoradiotherapy in potentially resectable oesophageal adenocarcinoma patients. Investigated dose levels range from $120 \mathrm{mg} / \mathrm{m} 2$ to $340 \mathrm{mg} / \mathrm{m} 2$. Evofosfamide will be administered one week before the start of chemoradiotherapy (CROSS-regimen) and repeated weekly up to a total of six doses. PET/CT acquisitions with hypoxia tracer ${ }^{18} \mathrm{~F}-\mathrm{HX} 4$ will be made before and after the first administration of evofosfamide, allowing early assessment of changes in hypoxia, accompanied with blood sampling to measure hypoxia blood biomarkers. Oesophagectomy will be performed according to standard clinical practice. Higher grade and uncommon non-haematological, haematological, and post-operative toxicities are the primary endpoints according to the CTCAEv4.0 and Clavien-Dindo classifications. Secondary endpoints are reduction in hypoxic fraction based on ${ }^{18} \mathrm{~F}-\mathrm{HX} 4$ imaging, pathological complete response, histopathological negative circumferential resection margin (RO) rate, local and distant recurrence rate, and progression free and overall survival.

(Continued on next page)
\end{abstract}

\footnotetext{
* Correspondence: lien.vandevoorde@gmail.com

${ }^{\dagger}$ Equal contributors

'Department of Radiation Oncology (MAASTRO), GROW-School for Oncology and Developmental Biology, Maastricht University Medical Centre, Maastricht, The Netherlands

Full list of author information is available at the end of the article
}

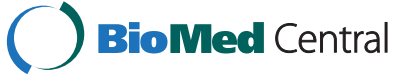

(c) 2016 The Author(s). Open Access This article is distributed under the terms of the Creative Commons Attribution 4.0 International License (http://creativecommons.org/licenses/by/4.0/), which permits unrestricted use, distribution, and reproduction in any medium, provided you give appropriate credit to the original author(s) and the source, provide a link to the Creative Commons license, and indicate if changes were made. The Creative Commons Public Domain Dedication waiver (http://creativecommons.org/publicdomain/zero/1.0/) applies to the data made available in this article, unless otherwise stated. 
(Continued from previous page)

Discussion: This is the first clinical trial testing evofosfamide in combination with chemoradiotherapy. The primary objective is to determine the dose limiting toxicity of this combined treatment and herewith to define the maximum tolerated dose and recommended phase 2 dose for future clinical studies. The addition of non-invasive repeated hypoxia imaging ('window-of-opportunity') enables us to identify the biologically effective dose. We believe this approach could also be used for other hypoxia targeted drugs.

Trial registration: ClinicalTrials.gov Identifier: NCT02598687.

Keywords: Oesophageal cancer, Neoadjuvant chemoradiotherapy, Evofosfamide, Oesophagectomy, Dose limiting toxicity, Hypoxia imaging, Window-of-opportunity trial

Abbreviations: ${ }^{18} \mathrm{~F}-\mathrm{HX} 4,\left[{ }^{18} \mathrm{~F}\right]-$ fluortanidazole; AUC, Target area under the concentration-time curve in $\mathrm{mg} / \mathrm{ml} / \mathrm{min}$; CAIX, Carbonic anhydrase IX; CRM, Circumferential resection margin; CROSS, ChemoRadiotherapy for Oesophageal cancer followed by Surgery Study; CT, Computed tomography; CTCAE, Common toxicity criteria for adverse events; DLT, Dose-limiting toxicity; ECG, Electrocardiogram; EUS, Endoscopic ultrasound; FDG, [ $\left[{ }^{18} \mathrm{~F}\right]$-fluordeoxyglucose; MTD, Maximum tolerated dose; nCRT, Neo-adjuvant chemoradiotherapy; OPN, Osteopontin; OS, Overall survival; PCR, Pathological complete remission; PET, Positron emission tomography; PICC, Peripherally inserted central catheter; RP2D, Recommended phase 2 dose; TH-302, Former abbreviation for evofosfamide; WHO, World Health Organisation

\section{Background}

The incidence of oesophageal cancer in developed Western countries has risen in recent decades [1]. Adenocarcinoma is now more prevalent than squamous cell carcinoma, with most tumours located in the distal oesophagus. A Western lifestyle is a risk factor and the disease is associated with obesity and symptomatic gastro-oesophageal reflux [2]. The standard treatment with curative intent for $\mathrm{T} 2$ or higher stage tumours consists of neoadjuvant chemoradiotherapy (nCRT) followed by surgery, [3] as confirmed by the Dutch ChemoRadiotherapy for Oesophageal cancer followed by Surgery Study (CROSS) [4]. In this study, significantly better 5-year overall survival (OS) rates were observed for patients treated with nCRT followed by surgery (47\%; $95 \%$ CI 39-54) when compared to surgery alone (33\%; $95 \%$ CI 26-40), with greater benefits for squamous cell carcinoma (61 \% vs. $30 \%$ ) than for adenocarcinoma (43\% vs. $33 \%$ ) [5]. However, little progress has been made in long-term survival (median OS $\sim 49$ months) and the patients' quality of life is still severely compromised by the impact of oesophagectomy. Therefore, there is an urgent need for new innovative treatment strategies.

The role of the tumour microenvironment in cancer progression, and especially the difference between this microenvironment and surrounding normal tissue, is a subject of increasing investigational interest with a specific focus on hypoxia. Hypoxic tumour cells promote a more aggressive phenotype, are associated with increased metastatic potential, and are known to be more resistant to standard chemoradiotherapy [6-14]. Recently, even micro-metastases have been shown to exhibit hypoxia [15]. Up to $5-10 \%$ of the oesophageal cancer patients suffer from progressive disease with metastases shortly after completion of neoadjuvant chemoradiotherapy [16] and the majority of patients eventually die because of metastatic disease. Therefore, hypoxia is an attractive target for newly developed drugs to increase the therapeutic effect of conventional oesophageal cancer treatment modalities.

Evofosfamide (TH-302) is a hypoxia-activated prodrug only activated under low levels of oxygen (hypoxia) [17-23]. Evofosfamide exploits the activation of a nitroimidazole prodrug by a process that involves the reduction of one electron, mediated by ubiquitous cellular reductases as the NADPH cytochrome P450 reductase to generate a radical anion prodrug. In the presence of oxygen (normoxia) the radical anion prodrug reacts rapidly with oxygen to produce superoxide and re-generate the original prodrug. Under the low-oxygen conditions of the hypoxic zones in tumours, however, the radical anion form of the prodrug has a longer half-life and can either fragment directly, or undergo further reductions, releasing the active drug bromo-isophosphoramide mustard that acts as a DNA cross-linker.

Recently, our group reported the radio-sensitizing effect of evofosfamide in a preclinical setting using syngeneic rat R1 rhabdomyosarcoma and human H460 NSCLC (non-small cell lung cancer) xenograft tumour models. Evofosfamide treatment significantly reduced the hypoxic fraction, by more than $80 \%$ compared to the control tumours in both tumour models. This was visualized at either micro-regional level or on PET images with the hypoxia tracer ${ }^{18} \mathrm{~F}-\mathrm{HX} 4$ (Fig. 1). Treatment with evofosfamide alone caused a significant delay in tumour growth while, when combined with radiotherapy (8 Gy), the growth delay was further enhanced. In addition, hypoxic fractions determined by pre-treatment ${ }^{18} \mathrm{~F}-\mathrm{HX} 4$ scans were predictive for the response associated with evofosfamide treatment. Therefore, a pre-treatment ${ }^{18} \mathrm{~F}-\mathrm{HX} 4$ scan 


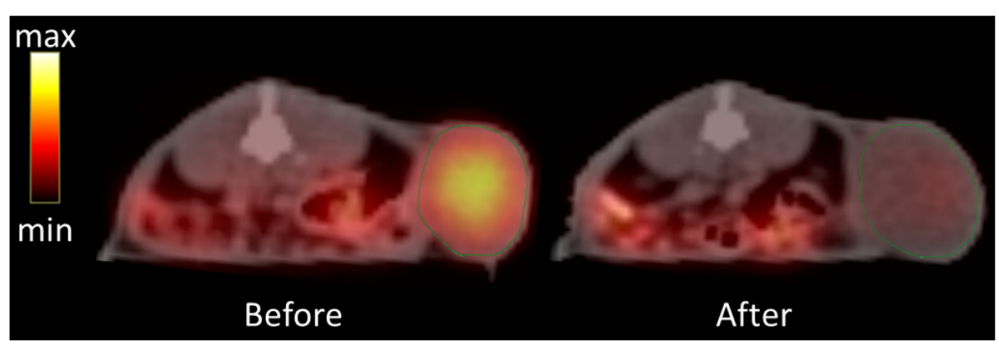

Fig. 1 Evofosfamide decreases the hypoxic fraction in a Rhabdomyosarcoma rat model. PET-scans with hypoxia tracer ${ }^{18} \mathrm{~F}-\mathrm{HX} 4$ were made before (day 0) and after (day 4) administering evofosfamide for four consecutive days at a dose of $25 \mathrm{mg} / \mathrm{kg}$

may be beneficial for selection of patients for evofosfamide treatment and a post treatment ${ }^{18} \mathrm{~F}-\mathrm{HX} 4$ scan enables to monitor treatment efficacy [20].

Evofosfamide has already been clinically investigated both as monotherapy and in combination with chemotherapy or other targeted cancer drugs in over 1,500 patients, and is currently under investigation in more than fifteen clinical trials registered at clinicaltrials.gov. Investigated tumours include soft tissue sarcoma, pancreatic cancer, non-small cell lung cancer, melanoma, and haematological malignancies. In general, the drug is well tolerated with main toxicities being higher-grade skin and mucosal toxicities, in particular in doses above $240 \mathrm{mg} / \mathrm{m}^{2}[24,25]$. In patients with advanced pancreatic cancer or soft-tissue sarcoma the combination of chemotherapy with evofosfamide achieved favourable outcomes, [26, 27] leading to two phase III clinical trials (NCT01746979 and NCT01440088). Despite the promising pre-clinical results demonstrating the potential added value of evofosfamide in combination with radiotherapy [20], to date no clinical studies have been performed to confirm this.

The primary objective of this $3+3$ dose escalation phase I 'window-of-opportunity' trial is to investigate the safety of evofosfamide in combination with the standard CROSS regimen in patients with distal oesophageal and oesophago-gastric junction adenocarcinoma, to determine the dose-limiting toxicities (DLTs) of the combined regimen and consequently to find the maximum tolerated dose (MTD) and recommended phase II dose (RP2D). As ${ }^{18} \mathrm{~F}-\mathrm{HX} 4$ has shown to be a hypoxia PET-tracer [28-35] with good repeatability in oesophageal cancer [36], two ${ }^{18} \mathrm{~F}-\mathrm{HX} 4$ PET-scans will be performed to characterize tumour hypoxia at baseline and visualize the potential change in hypoxia after the first administration of evofosfamide [9, 37].

\section{Methods/Design}

\section{Study design}

This is a phase I, non-randomized, single-centre, openlabel, $3+3$, 'window-of-opportunity' trial combining preoperative evofosfamide with the CROSS regimen (NCT02598687).

In this traditional $3+3$ dose-escalation design [38, 39], a cohort of three patients will enter a given dose level, and if no dose limiting toxicity (DLT) is observed 30 days after surgery, the trial will proceed to the next dose level. If a DLT occurs in 1 of 3 patients at a given dose level, 3 additional patients will be added to the same dose level cohort. If the occurrence of DLT remains limited to 1 out of 6 patients, the trial will proceed to the next dose level. If a DLT occurs in 2 or more patients at a certain dose level, dose escalation will be stopped. The previous dose level is then considered the maximum tolerated dose (MTD) and, therefore, the recommended dose for a phase II study. This is also summarized in Fig. 2. In this study three dose levels will be tested, which means that a maximum of nine to eighteen patients will be included.

\section{In- and exclusion criteria}

Prior to treatment, patients are discussed at the centralized multidisciplinary tumour board consisting of, inter alia, a surgeon, medical oncologist, nuclear medicine physician and radiation oncologist. Potentially curable patients with histologically proven stage IB-IIIC T2-4 distal oesophageal or oesophago-gastric junction adenocarcinoma are eligible to participate in this study. The minimum age is 18 years and the patients need to be fit for chemoradiotherapy with a normal baseline electrocardiogram (ECG) and a performance status of $0-2$ according to the World Health Organisation (WHO) classification.

The most important exclusion criteria are: a history of thoracic radiotherapy, recent severe cardiac or pulmonary disease, pregnancy, and/or viral infection.

\section{Study treatment}

All patients will receive $\mathrm{nCRT}$ according to the CROSS regimen (Carboplatin AUC $=2 \mathrm{mg} / \mathrm{ml} / \mathrm{min}$, Paclitaxel $50 \mathrm{mg} / \mathrm{m}^{2}$, concurrent radiotherapy $41.4 \mathrm{~Gy} / 23$ fractions) [4]. One starting dose of evofosfamide will be administered one week prior to the start of the nCRT, after which five additional administrations will be given 


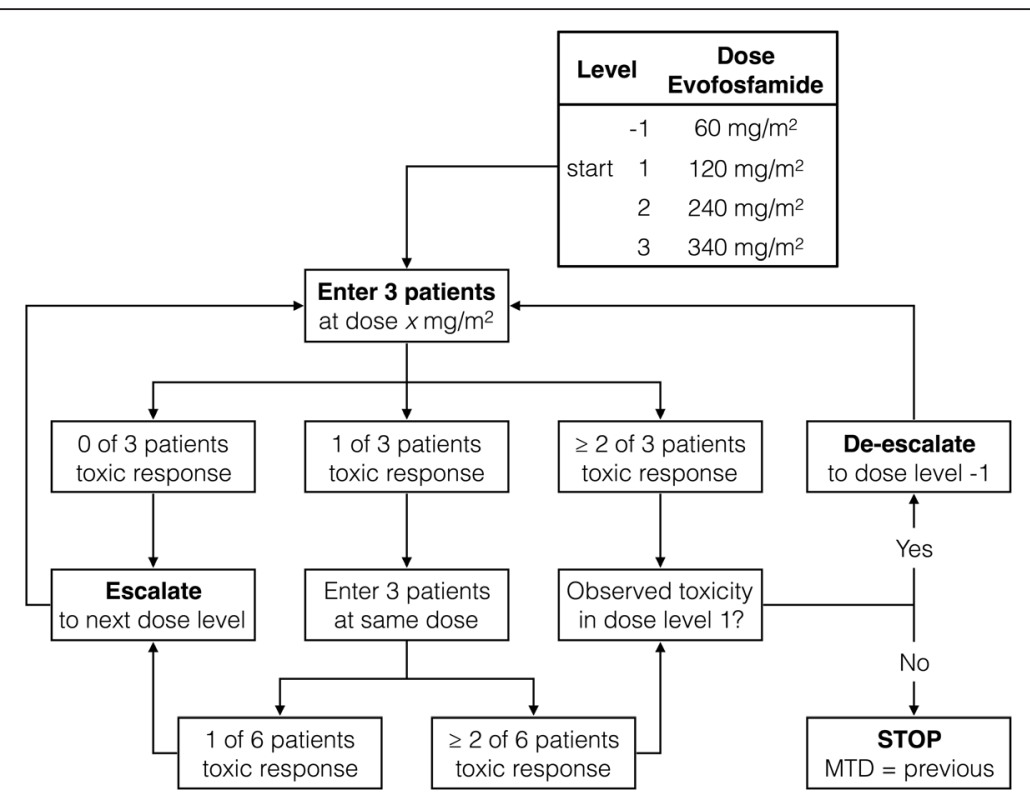

Fig. 2 Flowcharts summarizing the $3+3$ dose escalating study design. In the first cohort of patients evofosfamide will be administered at a dose of $120 \mathrm{mg} / \mathrm{m} 2$. Depending on the observed toxicity, we will escalate to dose level 2, or de-escalate to dose level -1. In further dose levels we can only escalate to the next dose (up to $340 \mathrm{mg} / \mathrm{m} 2$ ) or stop due to dose limiting toxicity

weekly on the same day as Carboplatin and Paclitaxel, but $2-4 \mathrm{~h}$ prior to the chemotherapeutics (Fig. 3). The drugs will be administered via a peripherally inserted central catheter (PICC) and an ECG will be made before and after administration of the first two fractions of evofosfamide. Investigated dose levels range from $120 \mathrm{mg} / \mathrm{m}^{2}$ to $340 \mathrm{mg} / \mathrm{m}^{2}$ evofosfamide, with the possibility to de-escalate to a dose level of $60 \mathrm{mg} / \mathrm{m}^{2}$ (Fig. 2). Due to the activation of evofosfamide under all hypoxic conditions, the patients will receive extra skin-care, e.g. by using cold packs during administration, to reduce hypoxia and prevent the possible occurrence of any severe skin-toxicities.

Surgical resection will be attempted six to ten weeks after completion of the nCRT, depending on the patient's characteristics and the lack of evidence for metastatic disease on a re-evaluation FDG-PET/CT-scan made before the planned surgery. Depending on tumour location and general comorbidity, either a minimally invasive transhiatal approach, including a one-field and low mediastinal lymph node dissection, or a transthoracic approach with a twofield lymph node dissection will be performed. The pathological tumour response of the resected specimen will be evaluated using the standardized pathology protocol, reporting, amongst others, the tumour regression grade according to the Mandard scoring [40], and the status of the resected lymph nodes and resection margins.

\section{Imaging}

Standard non-invasive diagnostic modalities include a (whole-body) FDG-PET/CT scan and/or endoscopic

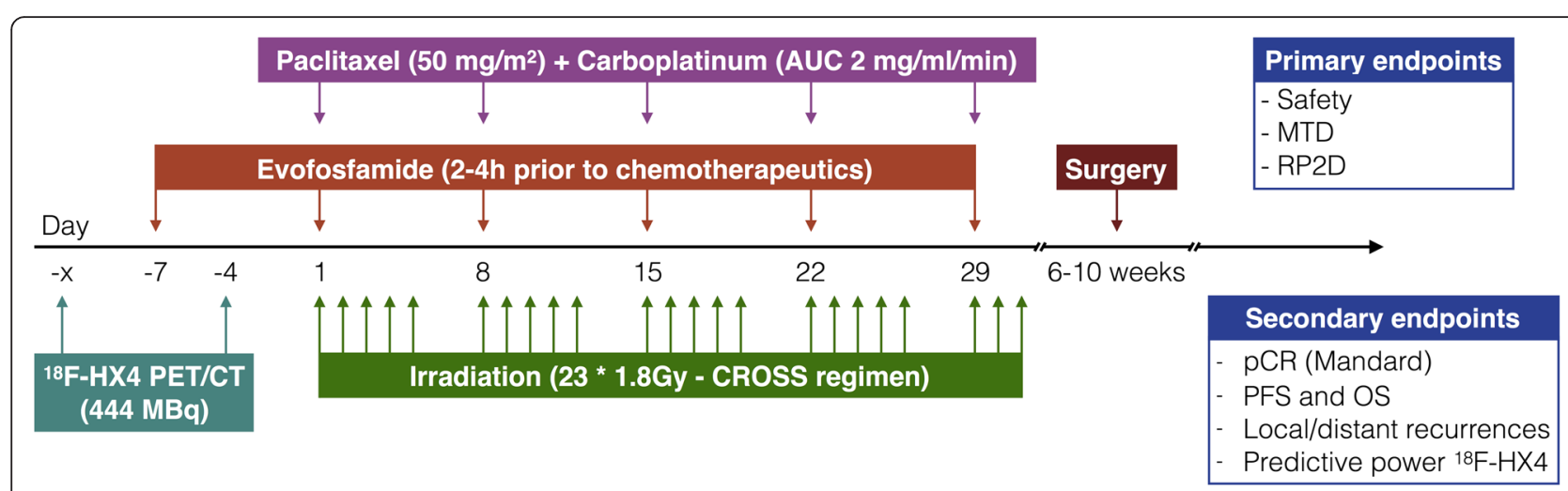

Fig. 3 Study treatment schedule. Prior to the start of the standard CROSS treatment, patients will receive additional ${ }^{18} \mathrm{~F}$-HX4 scans before and after the first dose of evofosfamide 
ultrasound (EUS) with biopsy. Patients that are included in this study will receive two additional PET/CT-scans with the hypoxia tracer ${ }^{18} \mathrm{~F}-\mathrm{HX} 4$ : the first scan at baseline and the second scan three days after the first administration of evofosfamide (Fig. 3). ${ }^{18} \mathrm{~F}-\mathrm{HX} 4$ will be administered via a bolus intravenous injection $444 \mathrm{MBq}(12 \mathrm{mCi})$, and the PET/CT-scan will be acquired $4 \mathrm{~h}$ post injection. The detailed acquisition protocol was previously described by Zegers et al. [33]. Based on the available diagnostic information, only a single bed position centred around the primary tumour site will be imaged and a slicethickness of $3 \mathrm{~mm}$ will be used for the CT-reconstruction $[28,29,33,34]$. On both days a blood sample (5-7 ml) will be drawn before administration of ${ }^{18} \mathrm{~F}-\mathrm{HX} 4$ to analyse the concentration of hypoxia related blood biomarkers carbonic anhydrase IX (CAIX) and osteopontin (OPN) [41].

\section{Study parameters and endpoints}

The main study endpoint is to determine the DLT and define the MTD and RP2D. DLT is defined as:

- Uncommon grade 3 or higher non-haematological toxicity according to the Common Terminology Criteria for Adverse Events (CTCAE) version 4.0. Grade III esophagitis in $50 \%$ of the patients is accepted.

- Grade 4 or higher haematological toxicity according to CTCAE version 4.0.

- Grade 4 or higher postoperative toxicity within 30 days post-surgery according to the Clavien-Dindo classification [42]. For anastomotic leakage and cardiorespiratory complication we accept a rate of $50 \%$ and $40 \%$ respectively.

- Any grade 2 or higher non-haematological toxicity that does not resolve to grade 0 or 1 toxicity by the start of the next cycle, which is considered a DLT according to the judgement of the investigator or sponsor.

- Inability to begin the next cycle of treatment within two weeks of the last dose due to unresolved toxicity.

Secondary endpoints include investigating the change in hypoxia based on ${ }^{18} \mathrm{~F}-\mathrm{HX} 4$ imaging and blood biomarkers to explore what would be the biologically effective dose, and anti-tumour activity measured by the rate of pathological complete response (pCR), histopathological negative circumferential resection margin (CRM) rate, local and distance recurrence rate, and progression free and overall survival.

\section{Post-treatment}

End of treatment is defined as the date of the last radiotherapy fraction in case of treatment completion according to protocol. When a patient drops-out before the end of treatment for any reasons other than DLT, he or she will be replaced by an additional patient.

If there is a complete remission on re-evaluation FDGPET/CT-scan after chemoradiotherapy, a patient can discuss the possibility for a wait-and-see strategy off protocol [43]. The decision to proceed to the next dose level of evofosfamide will be made when the minimum postsurgery or post-chemoradiotherapy (if no further surgery) follow-up of each patient in a particular dose level is 30 days.

Patients are examined weekly during the treatment. Follow-up starts directly after the end of treatment and adverse events will be assessed at 1 and 4 weeks after nCRT, right before surgery and one month after surgery. Thereafter, follow-up visits will be planned every three months in the first year after nCRT, twice in the second year, and then yearly until a minimum follow-up time of five years.

\section{Discussion}

\section{Known and potential risks and benefits}

The primary dose limiting toxicities of evofosfamide from clinical studies have indicated more haematological toxicity than in monotherapeutic chemotherapy. Skin and mucosal toxicities are common above doses of $240 \mathrm{mg} / \mathrm{m}^{2}$ $[24,25]$. The mucosal toxicities increase with dose but are still treatable with conservative approaches. The percentage of grade 3 esophagitis is expected to be higher in our proposed study design than with the standard CROSS treatment, but can be managed adequately (feeding tube, parenteral nutrition).

Evofosfamide has not been tested in combination with carboplatin, paclitaxel and radiotherapy before, so no pharmacological interactions between these drugs are known. A potential risk is that all three drugs can cause mild haematological toxicity (reversible leukopenia, neutropenia and/or lymphopenia) in some patients, but it is not known if this effect will be amplified by combining the drugs. Therefore we start with low dose levels of evofosfamide in comparison to the maximum tolerated doses in previous clinical studies. As an extra safety measure, patients in the first cohort will only be included when the previously included patient has finished chemoradiotherapy.

We are aware that with our trial design, combining evofosfamide with trimodality treatment, it will be difficult to determine the exact cause of potential adverse events. An alternative design would be to only include oesophageal cancer patients that receive either chemotherapy (carboplatin and/or paclitaxel) or radiotherapy. However, this strategy currently is only applied in very rare palliative cases. Moreover, the dose levels of chemotherapy and radiotherapy in a palliative setting are different 
than in a curative setting. Potentially curable patients will always receive trimodality therapy and withholding one of the treatment modalities to test the safety in combination with evofosfamide in potentially curable patients would, obviously, be very unethical, especially since the effectiveness of evofosfamide in oesophageal cancer is not proven yet. Hence, we believe that it is essential to test evofosfamide in the setting as proposed in this trial.

The benefit of this 'window-of-opportunity' trial is that the clinical activity of evofosfamide in patients with oesophageal cancer can be studied without being compromised by previous or interfering treatments [37]. Another benefit is that the combination therapy might overcome resistance to conventional treatment with chemoradiotherapy and creates a supra-additive effect with increased tumour response. Patients with a complete pathological response after neo-adjuvant treatment eventually could opt for a wait-and-see strategy to omit or postpone surgery.

\section{Explorative image analysis}

Hypoxia PET-tracer ${ }^{18} \mathrm{~F}-\mathrm{HX} 4$ has been extensively used at our institute in both pre-clinical and clinical studies. It is shown that the tracer is not associated with any toxicity [31], has a stable uptake pattern [33], provides complementary information to metabolic FDG imaging [34], and has a good spatial stability in lung, head and neck [29], and oesophageal cancer [36]. The design of this trial enables us to study the hypoxic response based on imaging biomarker ${ }^{18} \mathrm{~F}-\mathrm{HX} 4$ and blood biomarkers CAIX and OPN. This early response assessment will give us insight into the anti-tumour activity of evofosfamide, and can be used to define the optimal dose for future clinical research.

Calais et al. showed previously that high FDG-uptake regions at baseline identify tumour sub-volumes that are at a greater risk of recurrence [44]. Therefore explorative image analysis will be performed to visualize the spatial correlation between the baseline FDG-uptake and ${ }^{18} \mathrm{~F}$ HX4 uptake first, and later investigate if the high uptake regions correlate with the patterns of residual disease. Also the correlation between ${ }^{18} \mathrm{~F}$-HX4-imaging and hypoxia blood biomarkers CAIX and OPN will be evaluated.

Predictive models of outcome (e.g. pathological response, survival) will be developed based on a so-called Radiomics analysis. Radiomics is the extraction of a large number of quantitative intensity, shape and textural features from both CT and PET images [45]. It was shown that Radiomics features have prognostic value in both lung and head and neck cancer [46-48]. The additional value of Radiomics features in response prediction of oesophageal cancer patients is currently under investigation [49-51].
Another phase II clinical study currently investigates the effect of tumour hypoxia on the response to standard chemoradiation, by visualizing hypoxia with ${ }^{18} \mathrm{~F}-\mathrm{HX} 4$-imaging before treatment and two weeks after the start of treatment (NCT02584400). Since the HX4-scanning settings are identical, the imaging data of both studies provide complementary information about the behaviour and influence of tumour hypoxia in oesophageal cancer treatment. All of this together may be of additional value to better stratify patients in the future [52], by identifying patients who would benefit from hypoxia-selective treatment, such as evofosfamide, already in an early stage.

\section{Acknowledgements}

Authors acknowledge financial support from ERC advanced grant (ERC-ADG-2015, nº 694812 - Hypoximmuno) and the QulC-ConCePT project, which is partly funded by EFPI A companies and the Innovative Medicine Initiative Joint Undertaking (IMI JU) under Grant Agreement No. 115151. This research is also supported by the Dutch technology Foundation STW (grant $n^{\circ} 10696$ DuCAT \& n P14-19 Radiomics STRaTegy), which is the applied science division of NWO, and the Technology Programme of the Ministry of Economic Affairs. Authors also acknowledge financial support from EU 7th framework program (EURECA, ARTFORCE - $n^{\circ}$ 257144, REQUITE - $n^{\circ}$ 601826), SME Phase 2 (EU proposal 673780 - RAIL), the European Program H2020-PHC-2015 (BD2Decide - PHC30-689715), Kankeronderzoekfonds Limburg from the Health Foundation Limburg, Alpe d'HuZes-KWF (DESIGN) and the Dutch Cancer Society.

\section{Funding}

This trial is funded by the 2013 Atrium-Maastro grant, which is a collaboration between the former Atrium Medical Centre (since 2015 called Zuyderland Medical Centre) in Heerlen, and Maastro Clinic in Maastricht, The Netherlands.

\section{Authors' contributions}

$P L, L V, F W, F E, M S, W S, M N, M B, L D, W E$ and $R L$ are the investigators of this trial and have as such contributed to drafting the original study protocol, without which this manuscript could not have been written. LV, AC, MB and RL were responsible for writing, optimizing and submitting the study protocol. PL, MS, WE, WS were the PIs for the Atrium-Maastro grant application and designed the study. LV and RL wrote this manuscript. SP, KP and LD were responsible for the pre-clinical data. All authors read and approved the final manuscript.

\section{Authors' information}

Not applicable.

\section{Competing interests}

Threshold Pharmaceuticals supplies evofosfamide and ${ }^{18} \mathrm{~F}-\mathrm{HX} 4$. Threshold had no influence on the study protocol and was only contacted after the study protocol had been set. The authors declare that they have no competing interests.

\section{Consent for publication}

Not applicable.

\section{Ethics approval and consent to participate}

The study will be conducted according to the ICH Harmonised Tripartite Guideline for Good Clinical Practice and has been approved by the medical ethics committee of the Zuyderland Medical Centre (15 T85). All patients are given oral and written information about the study. They are given sufficient time to consider participation before the informed consent will be signed.

\section{Author details}

'Department of Radiation Oncology (MAASTRO), GROW-School for Oncology and Developmental Biology, Maastricht University Medical Centre, Maastricht, The Netherlands. ${ }^{2}$ Cancer Research UK \& Medical Research Council Oxford Institute for Radiation Oncology, Department of Oncology, University of Oxford, Oxford, UK. ${ }^{3}$ Department of Nuclear Medicine, Zuyderland Medical 
Centre, Sittard-Geleen/Heerlen, The Netherlands. ${ }^{4}$ Department of Pathology, Zuyderland Medical Centre, Sittard-Geleen/Heerlen, The Netherlands. ${ }^{5}$ Department of Medical Oncology, Zuyderland Medical Centre, Sittard-Geleen/Heerlen, The Netherlands. ${ }^{6}$ Department of Surgery, Zuyderland Medical Centre, Sittard-Geleen/Heerlen, The Netherlands. ${ }^{7}$ Surgical Collaborative Network Limburg, Limburg, The Netherlands.

\section{Received: 15 January 2016 Accepted: 11 August 2016}

\section{Published online: 17 August 2016}

\section{References}

1. Castro C, Bosetti C, Malvezzi M, Bertuccio P, Levi F, Negri E, et al. Patterns and trends in esophageal cancer mortality and incidence in Europe (1980-2011) and predictions to 2015. Ann Oncol. 2014;25:283-90.

2. Melhado RE, Alderson D, Tucker O. The Changing Face of Esophageal Cancer. Cancers (Basel). 2010;2:1379-404.

3. Tepper J, Krasna MJ, Niedzwiecki D, Hollis D, Reed CE, Goldberg R, et al. Phase III Trial of Trimodality Therapy With Cisplatin, Fluorouracil, Radiotherapy, and Surgery Compared With Surgery Alone for Esophageal Cancer : CALGB 9781. J Clin Oncol. 2008;26:1086-92.

4. van Hagen P, Hulshof MCCM, van Lanschot JJB, Steyerberg EW, van Berge Henegouwen MI, Wijnhoven BPL, et al. Preoperative chemoradiotherapy for esophageal or junctional cancer. N Engl J Med. 2012;366:2074-84.

5. Shapiro J, van Lanschot JJB, Hulshof MCCM, van Hagen $P$, van Berge Henegouwen MI, Wijnhoven BPL, et al. Neoadjuvant chemoradiotherapy plus surgery versus surgery alone for oesophageal or junctional cancer (CROSS): long-term results of a randomised controlled trial. Lancet Oncol. 2015;16:1090-8.

6. Bennewith KL, Dedhar S. Targeting hypoxic tumour cells to overcome metastasis. BMC Cancer. 2011;11:504.

7. Pettersen EO, Ebbesen P, Gieling RG, Williams KJ, Dubois L, Lambin P, et al. Targeting tumour hypoxia to prevent cancer metastasis. From biology, biosensing and technology to drug development : the METOXIA consortium. J Enzyme Inhib Med Chem. 2015;30:689-721.

8. Ebbesen P, Pettersen EO, Gorr TA, Jobst G, Williams K, Kieninger J, et al. Taking advantage of tumor cell adaptations to hypoxia for developing new tumor markers and treatment strategies. J Enzyme Inhib Med Chem. 2009;24:1-39.

9. Dubois $L J$, Niemans R, Van Kuijk SJA, Panth KM, Parvathaneni N, Peeters SGJA, et al. New ways to image and target tumour hypoxia and its molecular responses. Radiother Oncol. 2015;116:352-7.

10. Smits KM, Melotte V, Niessen HEC, Dubois L, Oberije C, Troost EGC, et al. Epigenetics in radiotherapy: Where are we heading ? Radiother Oncol. 2014;111:168-77.

11. Mengesha A, Dubois L, Chiu RK, Paesmans K, Wouters BG, Lambin P, Theys J. Potential and limitations of bacterial-mediated cancer therapy. Front Biosci. 2007;12:3880-91.

12. Wouters $B G$, Van Den Beucken T, Magagnin MG, Lambin P, Koumenis C. Targeting hypoxia tolerance in cancer. Drug Resist Updat. 2004;7:25-40.

13. Wouters BG, Koritzinsky M, Chiu RK, Theys J, Buijsen J, Lambin P. Modulation of Cell Death in the Tumor. Semin Oncol. 2003;13:31-41.

14. Wouters BG, Weppler SA, Koritzinsky M, Landuyt W, Nuyts S, Theys J, et al. Hypoxia as a target for combined modality treatments. Eur J Cancer. 2002;38:240-57.

15. Li X-F, O'Donoghue JA. Hypoxia in Microscopic Tumors. Cancer Lett. 2009;264:172-80

16. Stiekema J, Vermeulen D, Vegt E, Voncken FEM, Aleman BMP, Sanders J, et al. Detecting Interval Metastases and Response Assessment Using F-FDG PET / CT After Neoadjuvant Chemoradiotherapy for Esophageal Cancer. Clin Nucl Med. 2014;39:862-7.

17. Sun JD, Liu Q, Wang J, Ahluwalia D, Ferraro D, Wang Y, et al. Selective tumor hypoxia targeting by hypoxia-activated prodrug TH-302 inhibits tumor growth in preclinical models of cancer. Clin Cancer Res. 2012;18:758-70.

18. Hu J, Handisides DR, Van VE, De RH, Menu E, Vande BI, et al. Targeting the multiple myeloma hypoxic niche with $\mathrm{TH}-302$, a hypoxia-activated prodrug. Blood. 2010;116:1524-8.

19. Liapis V, Labrinidis A, Zinonos I, Hay S, Ponomarev V, Panagopoulos V, et al. Hypoxia-activated pro-drug TH-302 exhibits potent tumor suppressive activity and cooperates with chemotherapy against osteosarcoma. Cancer Lett. 2015;357:160-9.
20. Peeters SGJA, Zegers CML, Biemans R, Lieuwes NG, van Stiphout RGPM, Yaromina A, et al. TH-302 in combination with radiotherapy enhances the therapeutic outcome and is associated with pretreatment [18 F]HX4 hypoxia PET imaging. Clin Cancer Res. 2015;21:2984-93.

21. Saggar JK, Tannock IF. Activity of the hypoxia-activated pro-drug TH-302 in hypoxic and perivascular regions of solid tumors and its potential to enhance therapeutic effects of chemotherapy. Int J Cancer. 2014;134:2726-34.

22. Liu Q, Sun JD, Wang J, Ahluwalia D, Baker AF, Cranmer LD, et al. TH-302, a hypoxia-activated prodrug with broad in vivo preclinical combination therapy efficacy: optimization of dosing regimens and schedules. Cancer Chemother Pharmacol. 2012;69:1487-98.

23. Portwood S, Lal D, Hsu Y-C, Vargas R, Johnson MK, Wetzler M, et al. Activity of the hypoxia-activated prodrug, TH-302, in preclinical human acute myeloid leukemia models. Clin Cancer Res. 2013;19:6506-19.

24. Ganjoo KN, Cranmer LD, Butrynski JE, Rushing D, Adkins D, Okuno SH, et al. A Phase I Study of the Safety and Pharmacokinetics of the HypoxiaActivated Prodrug TH-302 in Combination with Doxorubicin in Patients with Advanced Soft Tissue Sarcoma. Oncology. 2011;80:50-6.

25. Weiss GJ, Infante JR, Chiorean EG, Borad MJ, Bendell JC, Molina JR, et al. Cancer Therapy : Clinical Phase 1 Study of the Safety, Tolerability, and Pharmacokinetics of TH-302, a Hypoxia-Activated Prodrug, in Patients with Advanced Solid Malignancies. Clin Cancer Res. 2011;17:2997-3005.

26. Chawla SP, Cranmer LD, Van Tine B a, Reed DR, Okuno SH, Butrynski JE, et al. Phase II Study of the Safety and Antitumor Activity of the HypoxiaActivated Prodrug TH-302 in Combination With Doxorubicin in Patients With Advanced Soft Tissue Sarcoma. J Clin Oncol. 2014;32:3299-306.

27. Borad MJ, Reddy SG, Bahary N, Uronis HE, Sigal D, Cohn AL, et al. Randomized Phase II Trial of Gemcitabine Plus TH-302 Versus Gemcitabine in Patients With Advanced Pancreatic Cancer. J Clin Oncol. 2015;33:1475-81.

28. Zegers CML, Van Elmpt W, Hoebers FJP, Troost EGC, Öllers MC, Mottaghy FM, Lambin P. Imaging of tumour hypoxia and metabolism in patients with head and neck squamous cell carcinoma. Acta Oncol (Madr). 2015;54:1378-84.

29. Zegers CML, Van Elmpt W, Szardenings K, Kolb H, Waxman A, Subramaniam RM, et al. Repeatability of hypoxia PET imaging using [18 F]HX4 in lung and head and neck cancer patients: a prospective multicenter trial. Eur J Nucl Med Mol Imaging. 2015;42:1840-9.

30. Wack L, Mönnich D, van Elmpt W, Zegers CML, Troost EGC, Zips D, Thorwarth D. HX4 for PET imaging of hypoxia - a simulation study. Acta Oncol (Madr). 2015;54:1370-7.

31. van Loon J, Janssen MHM, Ollers M, Aerts HJWL, Dubois L, Hochstenbag M, et al. PET imaging of hypoxia using [18 F]HX4: a phase I trial. Eur J Nucl Med Mol Imaging. 2010;37:1663-8.

32. Peeters SGJA, Zegers CML, Lieuwes NG, van Elmpt W, Eriksson J, van Dongen GAMS, et al. A Comparative Study of the Hypoxia PET Tracers [18 F]HX4, [18 F]FAZA, and [18 F]FMISO in a Preclinical Tumor Model. Int J Radiat Oncol. 2015;91:351-9.

33. Zegers CML, van Elmpt W, Wierts R, Reymen B, Sharifi H, Öllers MC, et al. Hypoxia imaging with $\left[{ }^{18} \mathrm{~F}\right] \mathrm{HX} 4$ PET in NSCLC patients: defining optimal imaging parameters. Radiother Oncol. 2013;109:58-64.

34. Zegers CML, van Elmpt W, Reymen B, Even AJG, Troost EGC, Ollers MC, et al. In Vivo Quantification of Hypoxic and Metabolic Status of NSCLC Tumors Using [18 F]HX4 and [18 F]FDG-PET/CT Imaging. Clin Cancer Res. 2014;20:6389-97.

35. Dubois $L$, Lieuwes NG, Janssen MHM, Peeters WJM, Windhorst AD. Preclinical evaluation and valication of [18 F]HX4, a promising hypoxia marker for PET imaging. Proc Natl Acad Sci. 2011;108:14620-5.

36. Klaassen R, Bennink RJ, van Tienhoven G, Bijlsma MF, Besselink MGH, van Berge Henegouwen MI, et al. Feasibility and repeatability of PET with the hypoxia tracer $[18 \mathrm{~F}] \mathrm{HX} 4$ in oesophageal and pancreatic cancer. Radiother Oncol. 2015;116:94-9.

37. Glimelius B, Lahn M. Window-of-opportunity trials to evaluate clinical activity of new molecular entities in oncology. Ann Oncol. 2011;22:1717-25.

38. Storer BE. Design and analysis of phase I clinical trials. Biometrics. 1989;45:3.

39. Le Tourneau C, Lee JJ, Siu LL. Dose escalation methods in phase i cancer clinical trials. J Natl Cancer Inst. 2009;101:708-20.

40. Mandard A-M, Dalibard F, Mandard J-C, Marnay J, Henry-Amar M, Pefiot J, et al. Pathologic Assessment of Tumor Regression after Preoperative Chemoradiotherapy of Esophageal Carcinoma Clinicopa thologic Correlations. Cancer. 1994;73:2680-6. 
41. Rademakers SE, Span PN, Kaanders JHAM, Sweep FCGJ, Van Der Kogel AJ, Bussink J. Molecular aspects of tumour hypoxia. Mol Oncol. 2008;2:41-53.

42. Clavien P, Barkun J, de Oliveira ML, Vauthey JN, Dindo D, Schulick RD, et al. The Clavien-Dindo Classification of Surgical Complications Five-Year Experience The Clavien-Dindo Classification of Surgical Complications. Ann Surg. 2009;250:187-96.

43. Noordman BJ, Shapiro J, Spaander MC, Krishnadath KK, van Laarhoven HW, van Berge Henegouwen Ml, et al. Accuracy of Detecting Residual Disease After Cross Neoadjuvant Chemoradiotherapy for Esophageal Cancer (preSANO Trial): Rationale and Protocol. JMIR Res Protoc. 2015;4:e79.

44. Calais J, Dubray B, Nkhali L, Thureau S, Lemarignier C, Modzelewski R, et al. High FDG uptake areas on pre-radiotherapy PET/CT identify preferential sites of local relapse after chemoradiotherapy for locally advanced oesophageal cancer. Eur J Nucl Med Mol Imaging. 2015;42:858-67.

45. Lambin P, Rios-Velazquez E, Leijenaar R, Carvalho S, van Stiphout RGPM, Granton $\mathrm{P}$, et al. Radiomics: extracting more information from medical images using advanced feature analysis. Eur J Cancer. 2012;48:441-6.

46. Leijenaar RTH, Carvalho S, Hoebers FJP, Aerts HJWL, van Elmpt WJC, Huang $\mathrm{SH}$, et al. External validation of a prognostic CT-based radiomic signature in oropharyngeal squamous cell carcinoma. Acta Oncol (Madr). 2015;54:1423-9.

47. Aerts HJWL, Velazquez ER, Leijenaar RTH, Parmar C, Grossmann P, Cavalho S, et al. Decoding tumour phenotype by noninvasive imaging using a quantitative radiomics approach. Nat Commun. 2014;5:4006.

48. Carvalho S, Leijenaar RTH, Velazquez ER, Oberije C, Parmar C, van Elmpt W, et al. Prognostic value of metabolic metrics extracted from baseline positron emission tomography images in non-small cell lung cancer. Acta Oncol (Madr). 2013:52:1398-404.

49. Hatt M, Majdoub M, Vallieres M, Tixier F, Cheze Le Rest C, Groheux D, et al. FDG PET uptake characterization through texture analysis: investigating the complementary nature of heterogeneity and functional tumor volume in a multi-cancer site patient cohort. J Nucl Med. 2015;56:38-44.

50. Hatt M, Visvikis D, Albarghach NM, Tixier F, Pradier O, Cheze-le RC. Prognostic value of 18 F-FDG PET image-based parameters in oesophageal cancer and impact of tumour delineation methodology. Eur J Nucl Med Mol Imaging. 2011;38:1191-202

51. Yip SSF, Coroller TP, Sanford NN, Huynh E, Mamon H, Aerts HJWL, Berbeco RI. Use of registration-based contour propagation in texture analysis for esophageal cancer pathologic response prediction. Phys Med Biol. 2016;61:906-22.

52. Lambin P, Zindler J, Vanneste B, Van De Voorde L, Jacobs M, Eekers D, et al. Modern clinical research: How rapid learning health care and cohort multiple randomised clinical trials complement traditional evidence based medicine. Acta Oncol (Madr). 2015;54:1289-300.

\section{Submit your next manuscript to BioMed Central and we will help you at every step:}

- We accept pre-submission inquiries

- Our selector tool helps you to find the most relevant journal

- We provide round the clock customer support

- Convenient online submission

- Thorough peer review

- Inclusion in PubMed and all major indexing services

- Maximum visibility for your research

Submit your manuscript at www.biomedcentral.com/submit

) Biomed Central 